\title{
Putting Patients Ahead by Leaving Nothing Behind: An Emerging Treatment Paradigm in Minimally Invasive Surgical Therapy for Benign Prostatic Hyperplasia
}

This article was published in the following Dove Press journal:

Medical Devices: Evidence and Research

\author{
Kevin T McVary' \\ Bilal Chughtai ${ }^{2}$ \\ Larry E Miller iD ${ }^{3}$ \\ Samir K Bhattacharyya ${ }^{4}$ \\ Ryan A Dornbier' \\ Dean $S$ Elterman ${ }^{5}$ \\ 'Center for Male Health, Department of \\ Urology, Stritch School of Medicine, \\ Loyola University Medical Center, \\ Maywood, IL, USA; ${ }^{2}$ Department of \\ Urology, Department of Obstetrics and \\ Gynecology, Weill Cornell Medicine, \\ New York, NY, USA; ${ }^{3}$ Department of \\ Biostatistics, Miller Scientific, Johnson \\ City, TN, USA; ${ }^{4}$ Health Economics \& \\ Market Access, Boston Scientific, \\ Marlborough, MA, USA; ${ }^{5}$ Division of \\ Urology, University Health Network, \\ Toronto, ON, Canada
}

\begin{abstract}
Lower urinary tract symptoms (LUTS) secondary to benign prostatic hyperplasia (BPH) are highly prevalent in older men. The long-term clinical utility of lifestyle modification and oral medications for LUTS is limited. There is a great clinical need for safe, effective, and durable BPH therapies for men who unsuccessfully attempt conservative measures. Enthusiasm for transurethral resection of the prostate has declined due to surgical risk, high rates of postoperative sexual dysfunction, and the perceived invasive nature therein. Consequently, interest has grown in developing minimally invasive surgical treatments (MISTs) that are efficacious but with a more favorable risk profile in order to better align with patient preferences. This review evaluates currently available MISTs for BPH. Further, we critically examine a "Leave Nothing Behind" philosophy in MIST for BPH since implantation of permanent metallic devices may be associated with increased long-term failure rates.
\end{abstract}

Keywords: benign prostatic hyperplasia, LUTS, minimally invasive surgical treatment, MIST, Rezum, UroLift

\section{Introduction}

Lower urinary tract symptoms (LUTS) are prevalent in older men and are responsible for a substantial clinical, societal, and economic global burden. ${ }^{1}$ With the continued aging of the population, the prevalence of LUTS is steadily increasing and now affects an estimated 1.5 billion men worldwide. ${ }^{2}$ The burden of bothersome LUTS increases with age, with moderate or severe symptoms reported in $12 \%$ of men aged 40-49 years and in 29\% of men aged 70-79 years. ${ }^{3}$ Affected men commonly experience hesitancy, straining, weak stream, and feeling of incomplete bladder emptying that significantly interferes with daily activities and compromises quality of life. While differential diagnosis of LUTS must consider precipitating factors such as diabetes mellitus, overactive bladder, urinary tract infection, and neurological disorders, urethral obstruction owing to prostatic tissue overgrowth is the most common etiology. This condition, benign prostatic hyperplasia (BPH), is a histologic diagnosis characterized by a proliferation of transition zone stromal and epithelial cells that encapsulate the urethra, narrow the luminal diameter, and restrict urinary flow.
Correspondence: Kevin T McVary Center for Male Health, Department of Urology, Stritch School of Medicine, Loyola University Medical Center, 2160 S, First Ave, Maywood, IL 60I53, USA

Email kmcvary@gmail.com
Medical Devices: Evidence and Research 202I:14 59-64 
A wide range of treatment options are available to men with LUTS, each of which have distinct advantages and limitations. Based on guidelines set forth by the American Urological Association (AUA), ${ }^{4}$ men with bothersome LUTS secondary to BPH should first consider lifestyle and behavioral modifications such as nightly fluid restriction, timed voiding, regular physical activity, and caffeine/alcohol limitations in order to delay symptom progression and avoid pharmacotherapy dependence. Men with persistent symptoms that interfere with daily activities despite conservative approaches are typically prescribed oral medications such as alpha-adrenergic antagonists, 5-alpha-reductase inhibitors, muscarinic receptor antagonists, beta-3 adrenergic agonists, or phosphodiesterase-5 inhibitors. While some patients derive lasting benefit, the long-term clinical utility of oral medications for LUTS may be limited since the majority of patients discontinue treatment in the first year owing to medication intolerance, side effects, or inadequate symptom relief. ${ }^{5-7}$ Consequently, there is a great clinical need for safe, effective, and durable BPH therapies for men who unsuccessfully attempt lifestyle modification and oral medication.

\section{Permanent Mechanical Implants for BPH MIST}

\section{Intraprostatic Stents}

The clinical use of an indwelling intraprostatic stent to provide physical retraction of obstructing prostatic tissue was first described in $19800^{8}$ Permanent intraprostatic stents are intended to provide immediate urethral patency with subsequent promotion of epithelialization and adherence to the prostatic stroma. While placing permanent metallic hardware in the prostatic urethra to improve luminal patency was initially an appealing concept, the clinical results with this therapy were unsatisfactory. In a 12-year follow-up study of men treated with the Urolume permanent prostatic stent, $82 \%$ required surgical device removal, mainly due to implant malpositioning, dislodgement, or irritative symptoms. ${ }^{9}$ Numerous other intraprostatic stent studies reported similar results where rates of urethral injury, infection, implant encrustation, device migration, and chronic pain were unacceptably high with resultant surgical reintervention rates of $38 \%$ to $70 \%{ }^{8,10,11}$ Although technological modifications were implemented in an effort to reduce complications and device failures, results with permanent mechanical intraprostatic stents remained clinically unacceptable and utilization of this therapy has since declined significantly. ${ }^{12}$

\section{UroLift}

The UroLift metallic implant (Teleflex, Wayne, PA) was approved for use in the United States in 2013. The UroLift procedure involves transurethral placement of permanent suture-metallic implants intended to mechanically separate obstructing prostatic lobes, restore urethral patency, and relieve LUTS. A number of studies including several randomized trials with mainly short-term follow-up durations have been performed with UroLift. ${ }^{13-23}$ Benefits of the UroLift procedure include quick postoperative resolution of LUTS, avoidance of catheterization, and rapid return to activities of normal living. Yet despite the immediate benefits of UroLift, concerns regarding device failures and associated surgical reoperations and medication restarts after UroLift treatment are beginning to mount as longer-term clinical data are becoming available.

In the UroLift pivotal trial, Roehrborn et a ${ }^{19}{ }^{\text {reported that }}$ $32(22.9 \%)$ of 140 men treated with UroLift required a surgical reintervention within 5 years. When accounting for patient dropouts over that 5-year period, the estimated surgical reintervention rate was $28.9 \% .{ }^{24} \mathrm{~A}$ significant number of these procedures were performed solely to remove encrusted or misplaced hardware from the prostate (Figure 1) -identical reasons for the failures of intraprostatic stents. A recent meta-analysis of 11 UroLift studies ${ }^{13-23}$ with over 2000 patients supported this finding showing the annual surgical reintervention rate was $6 \%$ per year $(\sim 30 \%$ over 5 years) (Figure 2) ${ }^{24}$ Another study reported that the treatment failure rate (patients who required surgical retreatment, medical retreatment, or experienced inadequate symptom relief) was $48 \%$ at 4 years with UroLift (Figure 3) ${ }^{25}$ Lastly, although many have reported that the UroLift procedure preserves sexual function, this claim is also coming under scrutiny. The bulk of UroLift studies utilize idiosyncratic adverse event definitions such as "new-onset and sustained ED" which may result in under-reporting of complication rates. When reviewing UroLift papers that reported adverse events using accepted regulatory definitions (eg the proportion of patients reporting a complication without mandating very specific conditions), sexual dysfunction with UroLift was more prevalent than previously thought with erectile dysfunction rates up to $11 \%,{ }^{23}$ important declines in erectile function in $8 \%,{ }^{26}$ and ejaculatory dysfunction in $9 \%$ of the patients. ${ }^{27}$ Overall, based on long-term best-evidence data, the clinical failure rate with the UroLift device appears to be 


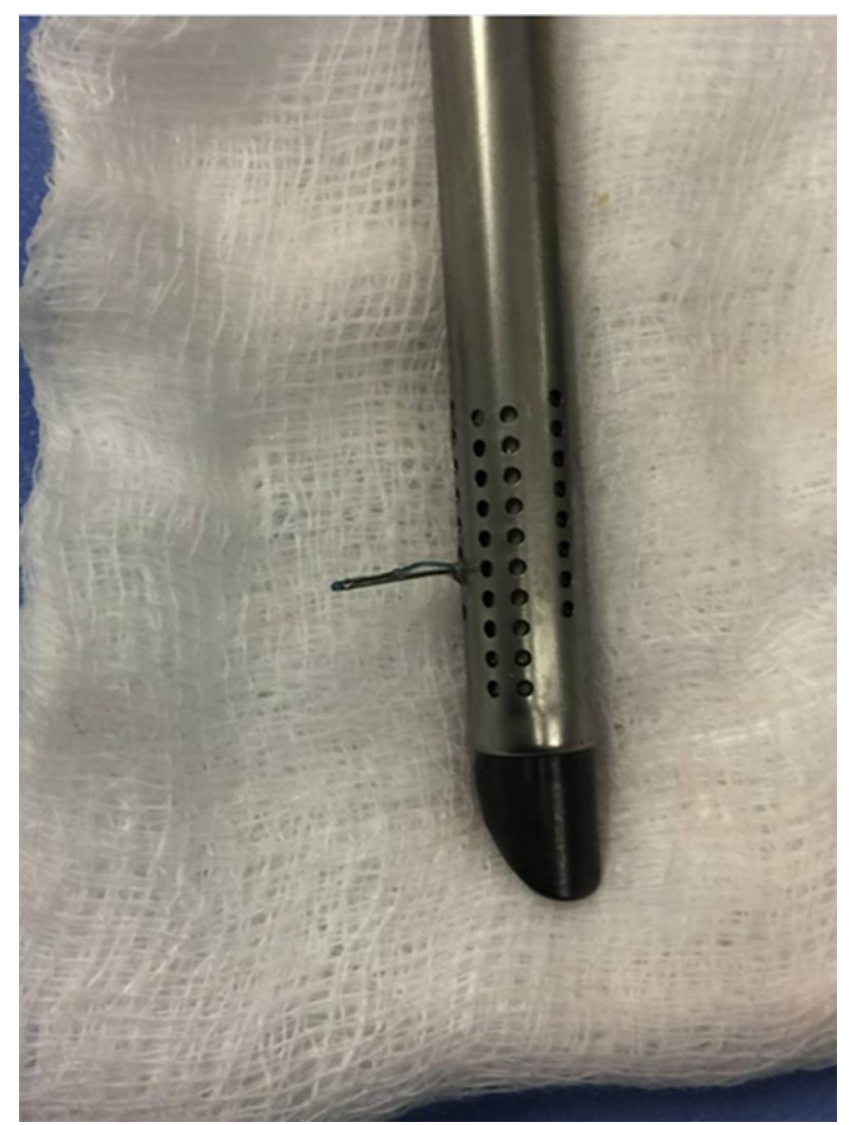

Figure I Failed UroLift implant caught in outer sheath of laser resectoscope during reoperation.

Notes: Reprinted from Urology Case Reports, Vol I6, Iqbal M, Jones R, Hughes S, et al, Low power HOLEP after failed UroLift: a case report using 50 Watt laser, Pages No. I I4-I I5, Copyright (2018), with permission from Elsevier. ${ }^{34}$

higher than previously reported based on the significant proportion of patients requiring surgical or medical retreatment.

Overall, the cumulative evidence shows that permanent metallic implants is not a risk-free treatment for LUTS/ BPH. While patients may realize short-term benefits, implant durability is questionable. Additionally, there are other hypothetical risks with permanent metallic implants that warrant further investigation. Most notably is the potential for the steel strut in the UroLift device to degrade image quality during multiparametric magnetic resonance imaging for prostate cancer diagnosis. Additionally, there is potential risk of complications in patients with allergy to nickel, titanium, or stainless steel. These findings highlight potential risks of utilizing permanent implants within a progressively expanding prostate gland and serve as the impetus to explore a "Leave Nothing Behind" treatment approach in BPH MIST where implantation of permanent metallic devices is discouraged.

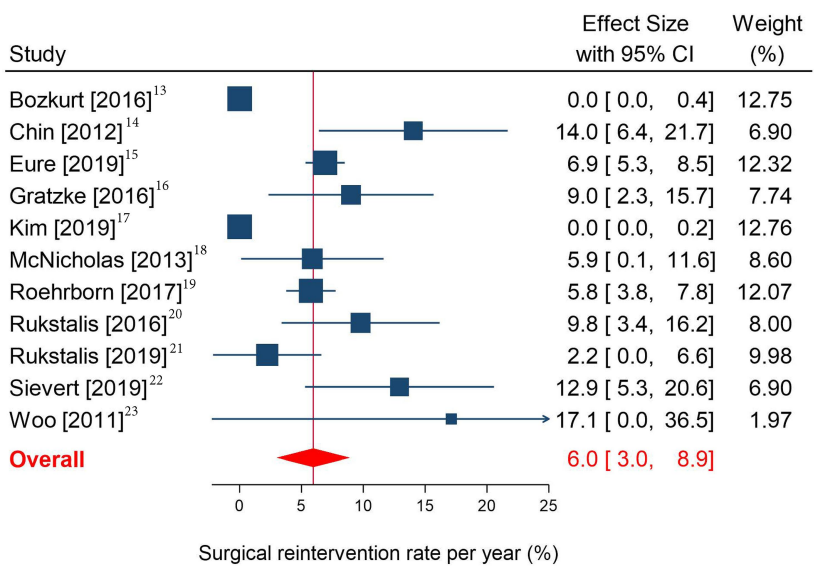

Figure 2 Annual surgical reintervention rate with UroLift.

Notes: The annual surgical reintervention rate and $95 \%$ confidence interval are plotted for each study. The size of the square is proportional to the study weight. The pooled rate among studies is denoted by the vertical line through the diamond apex. The $95 \%$ confidence interval is denoted by the diamond width. Heterogeneity: $I^{2}=99 \%, p<0.001$. Reproduced with permission from Miller LE, Chughtai B, Dornbier RA, et al. Surgical reintervention rate after prostatic urethral lift: systematic review and meta-analysis involving over 2000 patients. J Urol. 2020;204(5):1019-1026. Copyright (C) 2020, Wolters Kluwer Health. ${ }^{24}$

\section{The Rationale for BPH MIST}

As it relates to patients with BPH who failed to achieve satisfactory symptom relief with lifestyle changes or oral medications, transurethral resection of the prostate (TURP) is considered the gold standard treatment since this surgery provides clinically meaningful and durable relief from LUTS. ${ }^{4,28}$ However, TURP suffers as a gold standard when considering net health outcome

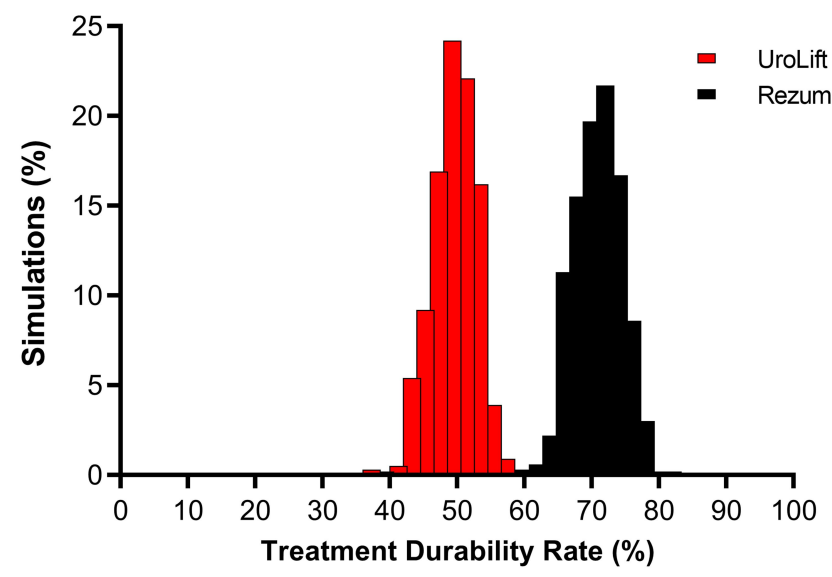

Figure 3 Monte Carlo simulations demonstrating the uncertainty in the estimation of treatment durability rates with minimally invasive $\mathrm{BPH}$ treatments.

Notes: The mean treatment durability rate through 4 years is $72 \%$ (95\% credible interval: $66 \%$ to $78 \%$ ) with WVTT and $51 \%$ (95\% credible interval: $45 \%$ to $56 \%$ ) with UroLift. Reproduced with permission from Miller LE, Te AE, Bhattacharyya SK, et al. A novel metric fortreatment durability in clinical trials of minimally invasive treatmentsfor benign prostatic hyperplasia. Expert Rev Med Devices. 2020;17(4):365369. () 2020 The Author(s). Published by Informa UK Limited, trading as Taylor \& Francis Group. ${ }^{25}$

Abbreviations: $\mathrm{BPH}$, benign prostatic hyperplasia; PUL, prostatic urethral lift; WVTT, water vapor thermal therapy. 
balancing efficacy, morbidity, and patient experience. The key benefits of TURP are offset by several major drawbacks including the need for regional/general anesthesia and hospitalization, increased anesthetic risk in older patients with coexisting medical conditions, and high rates of postoperative sexual dysfunction. ${ }^{29}$ Alternatives to TURP which are not in the MIST category and that utilize laser energy for destruction of prostate tissue such as holmium laser enucleation of the prostate or photoselective vaporization of the prostate are available yet postoperative complication and sexual dysfunction rates remain a concern with these tissue-removing invasive procedures. Consequently, interest has grown in developing MISTs, an entirely new category of treatments, that are similarly efficacious, provide a more favorable risk profile, and better align with patient preferences that increasingly place greater value on factors such as lower perioperative complication rates, faster recovery, and preservation of erectile and ejaculatory function.

\section{Rezum: A Leave Nothing Behind BPH MIST}

Approved by the FDA in 2015 and also recommended for use by the AUA, ${ }^{4,28}$ water vapor thermal therapy or
Rezum (Boston Scientific, Marlborough, MA) is an effective treatment for men with LUTS who wish to preserve sexual function, with prostates under $80 \mathrm{cc}$ in volume with or without an obstructive middle lobe. Rezum utilizes convective radiofrequency to create stored thermal energy in the form of steam, which is delivered transurethrally into the transition zone of the prostate to ablate tissue, thereby reducing LUTS. Because there is no permanent metallic hardware left in the body, there is no risk of mechanical device failure, device infection, or implant migration like with mechanical technologies.

A recent meta-analysis of Rezum for BPH reported that all measured clinical outcomes including the International Prostate Symptom Score (IPSS), IPSS-Quality of Life (IPSS-QOL), Benign Prostatic Hyperplasia Impact Index (BPHII), and maximum flow rate (Qmax) significantly improved following treatment and the changes from baseline were not only statistically significant but also clinically important at all follow-up intervals through 4 years. ${ }^{30}$ The treatment benefit of Rezum exceeded established minimally clinically important differences by 3 - to 4-fold for IPSS, 4- to 5-fold for IPSS-QOL, 6- to 8-fold for

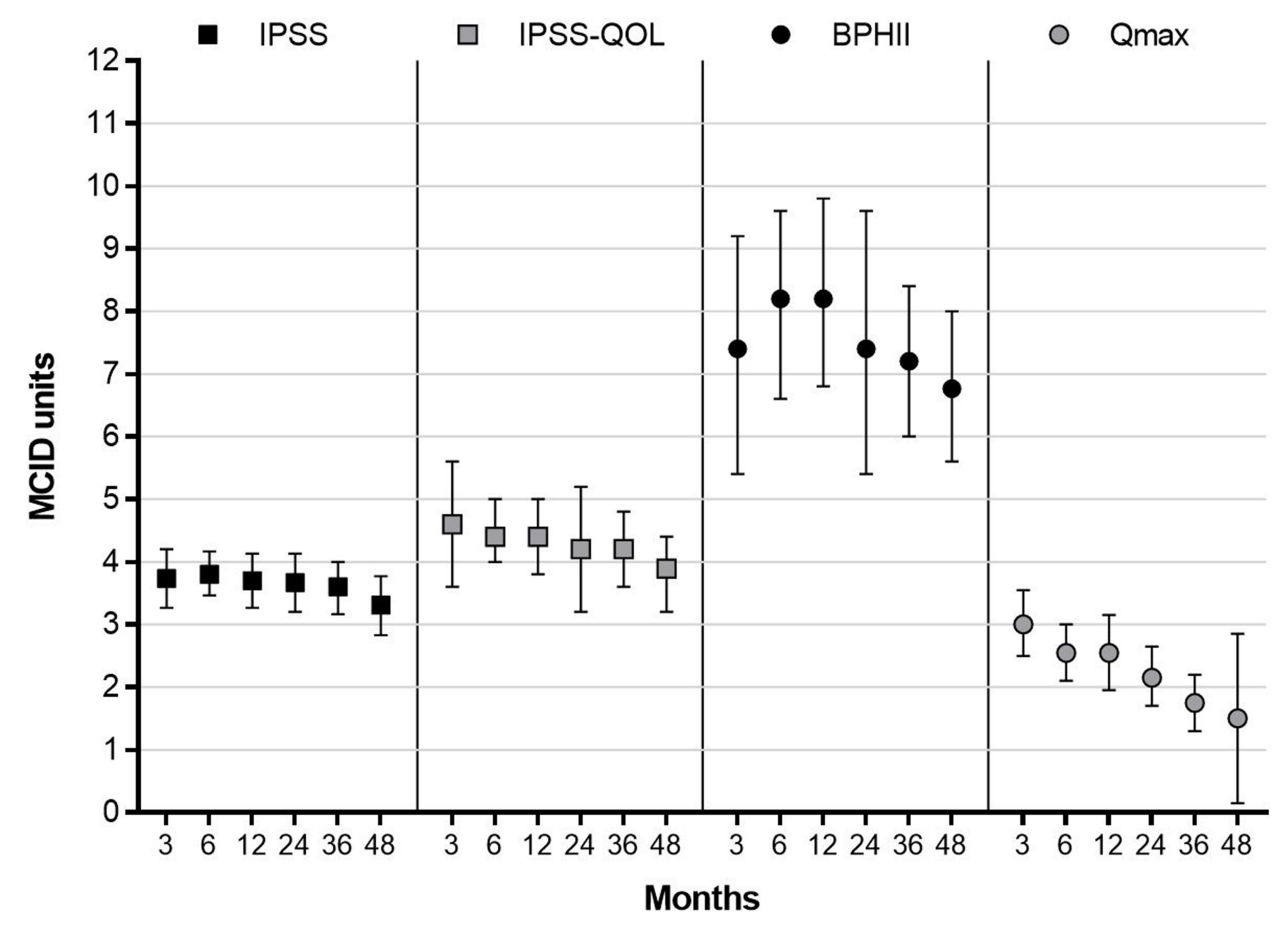

Figure 4 Improvement in BPH symptoms from baseline following water vapor thermal therapy reported in standardized MCID units with $95 \%$ confidence interval. Notes: The MCID is -3.0 for IPSS, ${ }^{35}-0.5$ for IPSS-QOL, ${ }^{35}-0.5$ for BPHII, ${ }^{35}$ and $2 \mathrm{~mL} / \mathrm{sec}$ for Qmax. ${ }^{36}$ Treatment effects below $0.5 \mathrm{MCID}$ units indicate that it is unlikely that an appreciable number of patients will show a clinically important benefit, treatment effects between 0.5 and I MCID units indicate that a treatment may be beneficial to an appreciable number of patients, and treatment effects above I MCID unit indicate that many patients may gain important benefits from treatment. ${ }^{37,38}$ Reproduced with permission from Miller LE, Chughtai B, McVary K, et al. Water vapor thermal therapy for lower urinary tract symptoms secondary to benign prostatic hyperplasia: systematic review and meta-analysis. Medicine. (Baltimore). 2020;99:e21365. Copyright (C 2020, Wolters Kluwer Health.. ${ }^{30}$

Abbreviations: BPH, benign prostatic hyperplasia; BPHII, Benign Prostatic Hyperplasia Impact Index; IPSS, International Prostate Severity Score; IPSS QOL, International Prostate Severity Score Quality of Life; MCID, minimal clinically important difference; Qmax, maximum flow rate. 
BPHII, and 2- to 3-fold for Qmax (Figure 4). Ejaculatory dysfunction occurred in $2 \%$ of the patients and there were no reports of de novo erectile dysfunction, indicating that Rezum durably preserved sexual function. Further evidence of the durability of Rezum comes from the results of a randomized trial where the severity of LUTS was reduced by approximately $50 \%$ over 5 years of follow-up and the surgical reintervention rate was only $4.4 \% .{ }^{31}$ Compared to intraprostatic stents with surgical reintervention rates of $38 \%$ to $82 \%{ }^{8-11}$ and UroLift with 5 -year surgical reintervention rates of approximately $29 \%,{ }^{24}$ the surgical reintervention rate with Rezum is very favorable. When additionally accounting for patients who attained clinically important improvements in symptom severity without medical or surgical retreatment, treatment durability rates are substantially higher with Rezum compared to UroLift ( $72 \%$ vs $52 \%){ }^{25}$ There are also certain disadvantages of Rezum. Catheterization for a mean of 3 days after the procedure has been reported, irritation of the urethra/ bladder may persist for 2-4 weeks in certain patients, and symptom resolution may take several weeks to occur. Overall, Rezum represents a distinct improvement in technological advancement and treatment durability for $\mathrm{BPH}$ patient care using an approach that leaves no permanent hardware behind.

\section{Evidence Interpretation}

LUTS secondary to BPH adversely impacts quality of life in many men. ${ }^{32}$ Men with persistent bothersome symptoms are often reluctant to undergo surgery due to concerns regarding procedural complications and sexual side effects. Mechanical treatments such as intraprostatic stents and UroLift may suffer from higher failure rates and poor long-term durability, albeit via different mechanism of action. The emerging long-term rates of surgical reintervention with UroLift as well as complications reported in real-world use are considerably higher than previously thought. In the American Urological Association guidelines for surgical management of LUTS attributed to $\mathrm{BPH},{ }^{4,28}$ Rezum and UroLift were recommended for well-selected men who desired preservation of sexual function. Yet Rezum is the only "Leave Nothing Behind MIST" and its utilization is supported by robust clinical evidence and with very low retreatment rates.

Clinical practice guidelines acknowledge the importance of patient preferences in determining the appropriate treatment for $\mathrm{BPH}$ and patients clearly prefer therapies that impact long-term disease progression over those that provide only short-term symptom improvements. ${ }^{33}$ Prospective studies that directly compare short- and longterm outcomes with these treatments while adequately controlling for potential confounding variables are encouraged to derive more definite conclusions regarding the comparative risks and benefits of each therapy. We encourage development of interventional algorithms that consider patient characteristics, patient preferences, and risk/ benefit profiles to assist in directing the patient and provider towards the single therapy that might be most suitable.

\section{Disclosure}

Kevin $\mathrm{T}$ McVary report grants and personal fees from Boston Scientific, personal fees from MedeonBio, grants from NIDDK, PI for Astellas, consultancy for Olympus LLC, outside the submitted work.

Bilal Chughtai reports consultancy for Boston Scientific, Olympus, and MediTate, outside the submitted work.

Larry E Miller is an employee of Miller Scientific and reports personal fees from Boston Scientific, during the conduct of the study.

Samir K Bhattacharyya reports being employed by Boston Scientific Corporation, outside the submitted work.

Dean S Elterman report grants and personal fees from Boston Scientific, during the conduct of the study.

The authors report no other potential conflicts of interest for this work.

\section{References}

1. Lee SWH, Chan EMC, Lai YK. The global burden of lower urinary tract symptoms suggestive of benign prostatic hyperplasia: A systematic review and meta-analysis. Sci Rep. 2017;7(1):7984. doi:10.1038/ s41598-017-06628-8

2. Irwin DE, Kopp ZS, Agatep B, et al. Worldwide prevalence estimates of lower urinary tract symptoms, overactive bladder, urinary incontinence and bladder outlet obstruction. BJU Int. 2011;108(7):11321138. doi:10.1111/j.1464-410X.2010.09993.x

3. Chute CG, Panser LA, Girman CJ, et al. The prevalence of prostatism: a population-based survey of urinary symptoms. J Urol. 1993;150:8589.

4. Foster HE, Barry MJ, Dahm P, et al. Surgical management of lower urinary tract symptoms attributed to benign prostatic hyperplasia: AUA guideline. J Urol. 2018;200:612-619.

5. Cindolo L, Pirozzi L, Fanizza C, et al. Drug adherence and clinical outcomes for patients under pharmacological therapy for lower urinary tract symptoms related to benign prostatic hyperplasia: populationbased cohort study. Eur Urol. 2015;68:418-425.

6. Koh JS, Cho KJ, Kim HS, et al. Twelve-month medication persistence in men with lower urinary tract symptoms suggestive of benign prostatic hyperplasia. Int J Clin Pract. 2014;68:197-202.

7. Zabkowski T, Saracyn M. Drug adherence and drug-related problems in pharmacotherapy for lower urinary tract symptoms related to benign prostatic hyperplasia. J Physiol Pharmacol. 2018;69. 
8. Fabian KM. [The intra-prostatic "partial catheter" (urological spiral) (author's trans1)]. Urologe A. 1980;19:236-238.

9. Masood S, Djaladat H, Kouriefs C, et al. The 12-year outcome analysis of an endourethral wallstent for treating benign prostatic hyperplasia. BJU Int. 2004;94:1271-1274.

10. Ogiste JS, Cooper K, Kaplan SA. Are stents still a useful therapy for benign prostatic hyperplasia? Curr Opinion Urol. 2003;13(1):51-57. doi:10.1097/00042307-200301000-00009

11. Chiou RK, Chen WS, Akbari A, et al. Long-term outcome of prostatic stent treatment for benign prostatic hyperplasia. Urology. 1996;48(4):589-593. doi:10.1016/S0090-4295(96)00327-5

12. Peyton CC, Badlani GH. The management of prostatic obstruction with urethral stents. Can J Urol. 2015;22(Suppl 1):75-81.

13. Bozkurt A, Karabakan M, Keskin E, et al. Prostatic urethral lift: a new minimally invasive treatment for lower urinary tract symptoms secondary to benign prostatic hyperplasia. Urol Int. 2016;96(2):202206. doi:10.1159/000441850

14. Chin PT, Bolton DM, Jack G, et al. Prostatic urethral lift: two-year results after treatment for lower urinary tract symptoms secondary to benign prostatic hyperplasia. Urology. 2012;79(1):5-11. doi:10.1016/ j.urology.2011.10.021

15. Eure G, Gange S, Walter P, et al. Real-world evidence of prostatic urethral lift confirms pivotal clinical study results: 2-year outcomes of a retrospective multicenter study. J Endourol. 2019;33(7):576584. doi:10.1089/end.2019.0167

16. Gratzke C, Barber N, Speakman MJ, et al. Prostatic urethral lift vs transurethral resection of the prostate: 2-year results of the BPH6 prospective, multicentre, randomized study. BJU Int. 2017;119 (5):767-775. doi:10.1111/bju.13714

17. Kim JH, Lee KS, Kim TH. Evaluation of clinical outcomes of prostatic urethral lift for benign prostatic hyperplasia: an Asian population study. World J Mens Health. 2019;24.

18. McNicholas TA, Woo HH, Chin PT, et al. Minimally invasive prostatic urethral lift: surgical technique and multinational experience. Eur Urol. 2013;64(2):292-299. doi:10.1016/j.eururo.2013.01.008

19. Roehrborn CG, Barkin J, Gange SN, et al. Five year results of the prospective randomized controlled prostatic urethral L.I.F.T. study. Can J Urol. 2017;24:8802-8813.

20. Rukstalis D, Rashid P, Bogache WK, et al. 24-month durability after crossover to the prostatic urethral lift from randomised, blinded sham. BJU Int. 2016;118(Suppl 3):14-22. doi:10.1111/bju.13666

21. Rukstalis D, Grier D, Stroup SP, et al. Prostatic Urethral Lift (PUL) for obstructive median lobes: 12 month results of the MedLift Study. Prostate Cancer Prostatic Dis. 2019;22(3):411-419. doi:10.1038/ s41391-018-0118-x

22. Sievert K-D, Schonthaler M, Berges R, et al. Minimally invasive prostatic urethral lift (PUL) efficacious in TURP candidates: a multicenter German evaluation after 2 years. World J Urol. 2003;13 (7):1353-1360. doi:10.1007/s00345-018-2494-1

23. Woo HH, Chin PT, McNicholas TA, et al. Safety and feasibility of the prostatic urethral lift: a novel, minimally invasive treatment for lower urinary tract symptoms (LUTS) secondary to benign prostatic hyperplasia (BPH). BJU Int. 2016;96(1):82-88. doi:10.1111/j.1464-410X.2011.10 342.x
24. Miller LE, Chughtai B, Dornbier RA, et al. Surgical reintervention rate after prostatic urethral lift: systematic review and meta-analysis involving over 2000 patients. J Urol. 2020;204(5):1019-1026. doi:10.1097/JU.0000000000001132

25. Miller LE, Te AE, Bhattacharyya SK, et al. A novel metric for treatment durability in clinical trials of minimally invasive treatments for benign prostatic hyperplasia. Expert Rev Med Devices. 2020;17 (4):365-369. doi:10.1080/17434440.2020.1742699

26. Woo HH, Bolton DM, Laborde E, et al. Preservation of sexual function with the prostatic urethral lift: a novel treatment for lower urinary tract symptoms secondary to benign prostatic hyperplasia. $J$ Sex Med. 2012;9:568-575.

27. Shah BB, Tayon K, Madiraju S, et al. Prostatic urethral lift: does size matter? J Endourol. 2018;32:635-638.

28. Foster HE, Dahm P, Kohler TS, et al. Surgical management of lower urinary tract symptoms attributed to benign prostatic hyperplasia: AUA guideline amendment 2019. J Urol. 2019;202:592-598.

29. Rassweiler J, Teber D, Kuntz R, et al. Complications of transurethral resection of the prostate (TURP)-incidence, management, and prevention. Eur Urol. 2006;50:969-979.

30. Miller LE, Chughtai B, McVary K, et al. Water vapor thermal therapy for lower urinary tract symptoms secondary to benign prostatic hyperplasia: systematic review and meta-analysis. Medicine (Baltimore).2020;99:e21365.

31. McVary K, Roehrborn C. Five year results of the prospective randomized controlled trial of water vapor thermal therapy for treatment of lower urinary tract symptoms due to benign prostatic hyperplasia. $J$ Urol. 2020;203:e1021.

32. Speakman M, Kirby R, Doyle S, et al. Burden of male lower urinary tract symptoms (LUTS) suggestive of benign prostatic hyperplasia (BPH) - focus on the UK. BJU Int. 2015;115:508-519.

33. Emberton M. Medical treatment of benign prostatic hyperplasia: physician and patient preferences and satisfaction. Int J Clin Pract. 2010;64:1425-1435.

34. Iqbal M, Jones R, Hughes $\mathrm{S}$, et al. Low power HOLEP after failed UroLift: a case report using 50 Watt laser. Urol Case Rep. 2018;16:114-115.

35. Barry MJ, Williford WO, Chang Y, et al. Benign prostatic hyperplasia specific health status measures in clinical research: how much change in the American Urological Association symptom index and the benign prostatic hyperplasia impact index is perceptible to patients? J Urol. 1995;154:1770-1774.

36. National Institute for Health and Care Excellence. Lower urinary tract symptoms in men: management; Clinical guideline [CG97]; 2010; https://www.nice.org.uk/guidance/CG97. Accessed August 21, 2019 .

37. Johnston BC, Patrick DL, Thorlund K, et al. Patient-reported outcomes in meta-analyses-part 2: methods for improving interpretability for decision-makers. Health Qual Life Outcomes. 2013;11:211.

38. Johnston BC, Thorlund K, Schunemann HJ, et al. Improving the interpretation of quality of life evidence in meta-analyses: the application of minimal important difference units. Health Qual Life Outcomes. 2010;8:116.
Medical Devices: Evidence and Research

\section{Publish your work in this journal}

Medical Devices: Evidence and Research is an international, peerreviewed, open access journal that focuses on the evidence, technology, research, and expert opinion supporting the use and application of medical devices in the diagnosis, monitoring, treatment and management of clinical conditions and physiological processes. The identification of novel devices and optimal use of existing devices which will lead to improved clinical outcomes and more effective patient management and safety is a key feature of the journal The manuscript management system is completely online and includes a very quick and fair peer-review system. Visit http:// www.dovepress.com/testimonials.php to read real quotes from published authors. 